\title{
Experimental Evidence of Hydrodynamic Instantons: The Universal Route to Rogue Waves
}

\author{
Giovanni Dematteis $\odot,{ }^{1,2}$ Tobias Grafke, ${ }^{3}$ Miguel Onorato $\odot,{ }^{2,4}$ and Eric Vanden-Eijnden ${ }^{5}$ \\ ${ }^{1}$ Dipartimento di Scienze Matematiche, Politecnico di Torino, \\ Corso Duca degli Abruzzi 24, I-10129 Torino, Italy \\ ${ }^{2}$ Dipartimento di Fisica, Università degli Studi di Torino, Via Pietro Giuria 1, 10125 Torino, Italy \\ ${ }^{3}$ Mathematics Institute, University of Warwick, Coventry CV4 7AL, United Kingdom \\ ${ }^{4}$ INFN, Sezione di Torino, Via Pietro Giuria 1, 10125 Torino, Italy \\ ${ }^{5}$ Courant Institute, New York University, 251 Mercer Street, New York, New York 10012, USA
}

(Received 3 July 2019; revised manuscript received 2 October 2019; published 18 December 2019)

\begin{abstract}
A statistical theory of rogue waves is proposed and tested against experimental data collected in a long water tank where random waves with different degrees of nonlinearity are mechanically generated and free to propagate along the flume. Strong evidence is given that the rogue waves observed in the tank are hydrodynamic instantons, that is, saddle point configurations of the action associated with the stochastic model of the wave system. As shown here, these hydrodynamic instantons are complex spatiotemporal wave field configurations which can be defined using the mathematical framework of large deviation theory and calculated via tailored numerical methods. These results indicate that the instantons describe equally well rogue waves created by simple linear superposition (in weakly nonlinear conditions) or by nonlinear focusing (in strongly nonlinear conditions), paving the way for the development of a unified explanation to rogue wave formation.
\end{abstract}

DOI: 10.1103/PhysRevX.9.041057

\section{INTRODUCTION}

A fascinating phenomenon observed in a wide class of nonlinear dispersive systems is the occurrence of rogue waves with abnormally large amplitude; they are found in sea surface gravity waves [1,2], nonlinear fiber optics [3], plasmas [4], and Bose-Einstein condensates. Rogue waves have received a lot of attention in the past 20 years, and different mechanisms for their formation have been put forward, but a definite explanation has yet to be agreed upon [2,5-9]. To settle this question, studies in wave flumes or basins are interesting because they permit us to create and measure wave states by means of mechanical wave generators under controlled conditions meant to mimic (after rescaling) those in the sea. The water surface in the tank can be monitored accurately with high space-time resolution, and abundant statistics can be collected. In onedimensional experiments that mimic an idealized longcrested rescaled sea, if the surface is sufficiently energetic,

Published by the American Physical Society under the terms of the Creative Commons Attribution 4.0 International license. Further distribution of this work must maintain attribution to the author(s) and the published article's title, journal citation, and DOI.
Subject Areas: Fluid Dynamics, Nonlinear Dynamics, Statistical Physics nonlinear focusing effects take over linear dispersion and are known to be responsible for increasing the likelihood of the rogue waves. This leads to non-Gaussian fat-tailed statistics for their amplitude [2,10], as opposed to the Gaussian statistics observed in the dispersive regime.

In the present article, we propose a statistical theory of rogue waves and test it against experiments performed in the one-dimensional setting of the wave flume. We show that, in the full range of experimental conditions tested, the rogue waves we observe closely resemble hydrodynamic instantons [11-16]: these are specific spatio-temporal configurations of the wave field which we define within the framework of large deviation theory (LDT) as the minimizers of an action associated with the random wave model used to describe the system. Here we focus on the nonlinear Schrödinger equation (NLSE) with random initial data, but the approach is generalizable to more complicated models. The finding that instantons explain experimental rogue waves for a wide range of surface conditions in the tank is striking because it offers a unified description of these waves. In particular, our approach encompasses two of the main existing theories for rogue wave creation: (i) the theory of quasideterminism $[17,18]$, which predicts that the rogue wave is created by linear superposition effects and its shape is given by the 
autocorrelation function of the wave field, and (ii) the semiclassical theory $[19,20]$, which asserts instead that localized perturbations in the wave field can lead to the formation of a Peregrine soliton via nonlinear focusing instability. Our approach reconciles these two, apparently incompatible, theories and smoothly interpolates between them as the experimental control parameters are varied: when the nonlinear effects are weak, the shape of the instantons converges to the autocorrelation function predicted by the theory of quasi-determinism, and when the nonlinear effects are strong, their shape converges to that of the Peregrine soliton. Because the instanton calculus proposed in this paper uses as limiting parameter the maximal wave amplitude itself, without condition on model parameters or regimes in the NLSE, it allows us to assess the validity of the quasideterministic and semiclassical theories by comparing them to the results of our approach in appropriate regimes. Our approach could also be useful in the context of other nonlinear theories for rogue waves based on NLSE, like statistical approaches based on the Alber and the Wigner equations [21-26]. We also stress that the method proposed here can be generalized to the full two-dimensional setting, as well as other relevant physical systems where an understanding of extreme events is important $[27,28]$ but made challenging by the complexity of the models involved combined with the stochasticity of their evolution and the uncertainty of their parameters [27,29-32]. In this sense our approach adds to other rare events methods [33-40].

The remainder of this paper is organized as follows. We introduce the experimental setup in Sec. II. In Sec. III, we explain how we extract extreme event data from the experimental measurements. Our approach based on large deviation theory is presented in Sec. IV, where we also describe how we compute the instanton for the rogue waves. Theory and experiment are then compared in Sec. V, with special focus on the quasilinear and highly nonlinear limiting cases. We conclude in Sec. VI by discussing the implications of our results in the context of a unified theory of rogue waves.

\section{EXPERIMENTAL SETUP}

The experimental data were recorded in the 270-m-long wave flume at Marintek (Norway) [41,42], schematically represented in Fig. 1. At one end of the tank a plane-wave generator perturbs the water surface with a predefined random signal. These perturbations create long-crested wave trains that propagate along the tank toward the opposite end, where they eventually break on a smooth beach that suppresses most of the reflections. The water surface $\eta(x, t)$ is measured by probes placed at different distances from the wave maker ( $x$ coordinate). The signal at the wave maker $\eta(x=0, t) \equiv \eta_{0}(t)$ is prepared according to the stationary random-phase statistics with deterministic spectral amplitudes $C\left(\omega_{j}\right)$ :

$$
\eta_{0}^{e}(t)=\sum_{j=1}^{N} \sqrt{2 C\left(\omega_{j}\right) \delta \omega} \cos \left(\omega_{j} t+\phi_{j}\right) .
$$

Here the phases $\phi_{j}$ are mutually independent random variables uniformly distributed on $[0,2 \pi], \delta \omega=(2 \pi / \tau)$, $\omega_{j}=j \delta \omega$, and $\tau$ is the time-series length. This guarantees that, for $N$ and $\tau$ sufficiently large, $\eta_{0}^{e}(t)$ is approximately a stationary Gaussian random field with energy spectrum $C(\omega)>0$, i.e.,

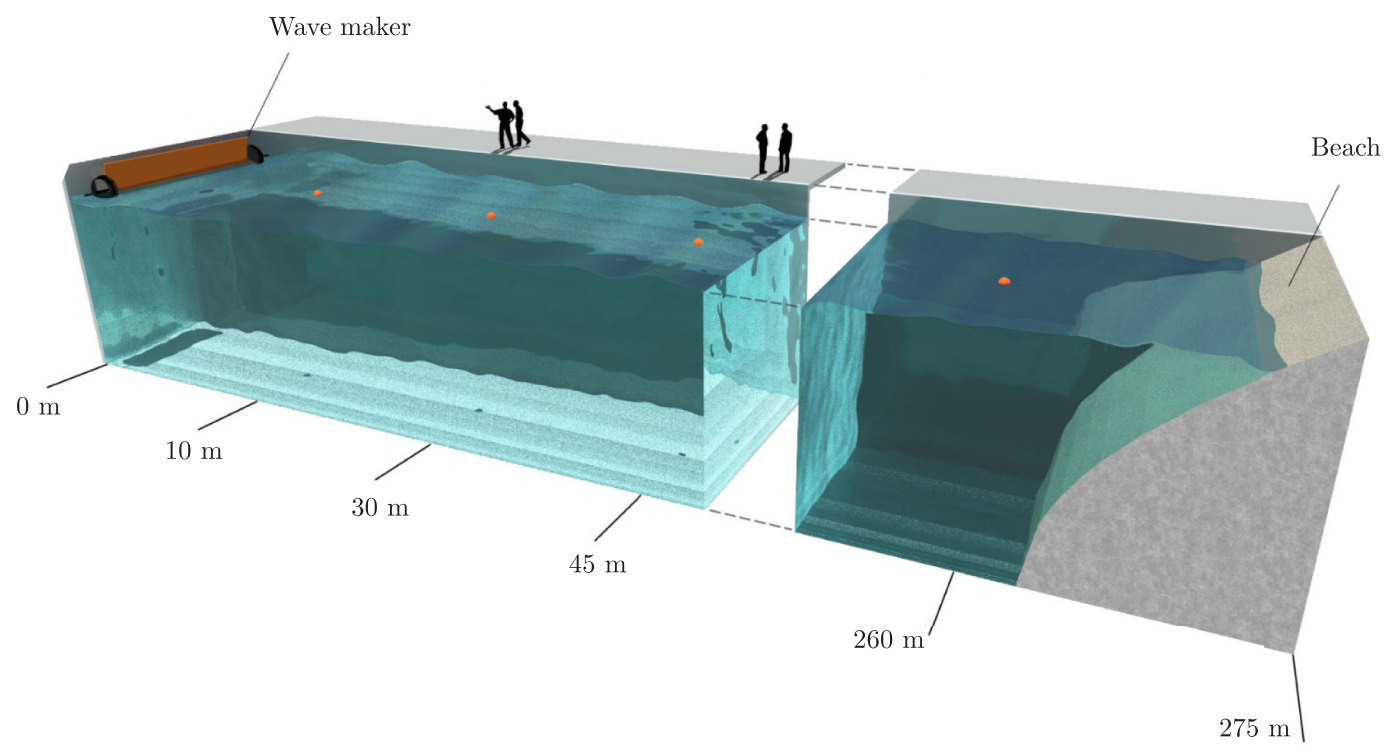

FIG. 1. Wave flume experiment. The wave maker generates a random wave field with stationary Gaussian statistics with the JONSWAP energy spectrum observed in the oceans. The planar wave fronts propagate along the water tank, where the surface elevation $\eta$ is measured by vertical probes. 


$$
\begin{aligned}
\left\langle\eta_{0}^{e}(t) \eta_{0}^{e}\left(t^{\prime}\right)\right\rangle & =\sum_{j=1}^{N} C\left(\omega_{j}\right) \delta \omega \cos \left[\omega_{j}\left(t-t^{\prime}\right)\right] \\
& \sim \int_{0}^{\infty} C(\omega) \cos \left[\omega\left(t-t^{\prime}\right)\right] d \omega
\end{aligned}
$$

where the bracket denotes expectation with respect to the random phases $\phi_{j}$. In the experiment, $C(\omega)$ is taken to be the Joint North Sea Wave Project (JONSWAP) spectrum [43] of deep water waves observed in the ocean,

$$
C(\omega)=\frac{\alpha g^{2}}{\omega^{5}} \exp \left[-\frac{5}{4}\left(\frac{\omega_{0}}{\omega}\right)^{4}\right] \gamma^{\exp \left[-\left(\omega-\omega_{0}\right)^{2} / 2 \sigma_{J}^{2} \omega_{0}^{2}\right]} .
$$

Here $g=9.81 \mathrm{~ms}^{-2}$ is the gravity acceleration, $\omega_{0}=$ $4.19 \mathrm{~s}^{-1}$ is the carrier frequency (spectral peak), and $\sigma_{J}=$ 0.07 if $\omega \leq \omega_{0}$ and $\sigma_{J}=0.09$ if $\omega>\omega_{0}$. These parameters are fixed for all sea states, and we can use the dispersion relation of surface gravity waves in deep water to obtain the carrier wave number $k_{0}=\omega_{0}^{2} / g=1.79 \mathrm{~m}^{-1}$. The remaining parameters $\alpha$ and $\gamma$ in Eq. (3) are dimensionless and vary according to weather conditions. In the experiments, $\alpha=0.012$ throughout, while the enhancement factor $\gamma$ ranges from 1 to 6 , which is a realistic range of values for the ocean measurements from calmer to rougher sea states. In the water waves community, it is common to introduce the significant wave height $H_{s}$, as a statistical measure of the average wave height, here defined as

$$
H_{s}=4 \sigma=4\left(\int_{0}^{\infty} C(\omega) d \omega\right)^{1 / 2},
$$

where $\sigma=\left\langle\eta_{0}^{2}\right\rangle^{1 / 2}$ is the standard deviation of the surface elevation, which both depend on $\gamma$ as well as the other parameters in Eq. (3) that we keep fixed as specified above. We also introduce a characteristic bandwidth $\Omega$ of the JONSWAP spectrum defined as

$$
\Omega=\text { width of } C(\omega) \text { at half height. }
$$

Experimental data were collected for three different regimes: quasilinear $\left(\gamma=1, H_{s}=0.11 \mathrm{~m}\right)$, intermediate

TABLE I. The relevant parameters in the three experimental regimes considered. The parameters $\gamma, H_{s}$, and $\Omega$ are used to characterize the JONSWAP spectrum enforced by the wave maker. The parameter $\epsilon$ is used to quantify the strength of nonlinear versus dispersive effects in NLSE and is defined in Sec. IVA. The two lengths $L_{\text {lin }}$ and $L_{\text {Per }}$ measure the typical scales over which these effects occur: they are defined in Sec. V C and are useful for the interpretation of Fig. 6.

\begin{tabular}{llcccccc}
\hline \hline Regime & $\gamma$ & $H_{s}(\mathrm{~m})$ & $\Omega\left(\mathrm{s}^{-1}\right)$ & $\epsilon$ & $L_{\text {lin }}(\mathrm{m})$ & $L_{\mathrm{Per}}(\mathrm{m})$ \\
\hline Quasilinear & 1 & 0.11 & 2.12 & 0.15 & 8.9 & 32 \\
Intermediate & 3.3 & 0.13 & 0.90 & 1.13 & 46 & 61 \\
Highly nonlinear & 6 & 0.15 & 0.76 & 2.23 & 69 & 65 \\
\hline \hline
\end{tabular}

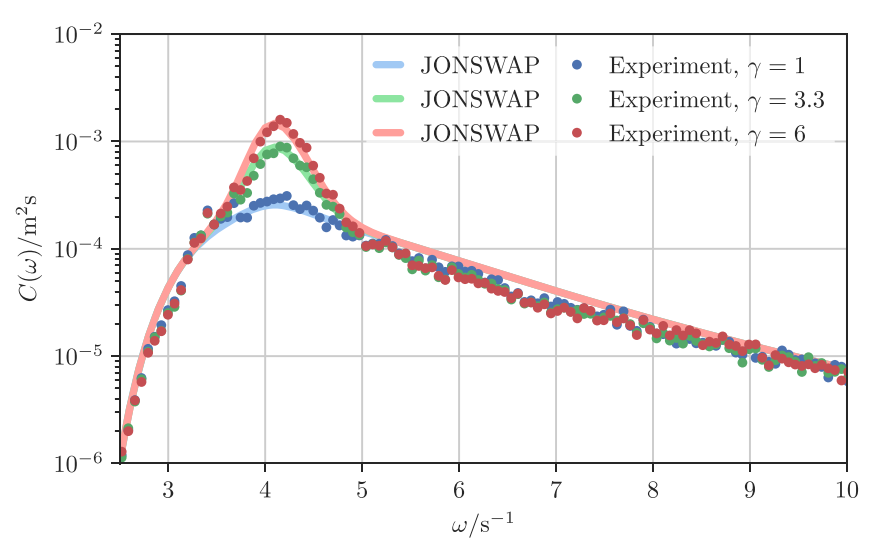

FIG. 2. JONSWAP spectra from Eq. (3) for the three experimental regimes of Table I (lines), compared to experimental measurements at the $x=10 \mathrm{~m}$ probe (dots). These spectra remain roughly constant through the tank, except for small changes that are the signature of non-Gaussian effects that develop [42].

$\left(\gamma=3.3, H_{s}=0.13 \mathrm{~m}\right)$, and highly nonlinear $(\gamma=6$, $H_{s}=0.15 \mathrm{~m}$ ); see Table I. Note that these three regimes have comparable significant wave heights $H_{s}$, but the difference in their enhancement factors $\gamma$ has significant dynamical consequences, as discussed in Sec. IV where we introduce and explain the additional parameters $\epsilon, L_{\text {lin }}$, and $L_{\mathrm{Per}}$ listed in the table. Experimental measurements of the spectrum for the three regimes are depicted in Fig. 2.

For each set, we use data from 5 time series, each of which is $25 \mathrm{~min}$ long. The surface elevation $\eta$ is measured simultaneously by 19 probes placed at different locations along the axes at the center of the tank, recording data with a rate of 40 measurements per second. At each of two different positions $(x=75 \mathrm{~m}$ and $x=160 \mathrm{~m})$ two extra probes closer to the sides are used to check that the wave fronts remain planar.

\section{EXTREME-EVENT FILTERING: EXTRACTING ROGUE WAVES FROM EXPERIMENTAL DATA}

To characterize the dynamics leading to extreme events of the water surface, we adopt the following procedure: at a fixed location $x=L$ along the flume, we select small observation windows around all temporal maxima of $\eta$ that exceed a threshold $z$. The choice of the threshold $z$ is meant to select extreme events with a similar probability for all sets: the values of $z=H_{s}=4 \sigma$ for the quasilinear set, $z=$ $1.1 H_{s}=4.4 \sigma$ for the intermediate set, and $z=1.2 H_{s}=$ $4.8 \sigma$ for the highly nonlinear set lead, respectively, to 78 , 99 , and 88 registered events where the maximum of the surface elevation exceeds the threshold at the $45 \mathrm{~m}$ probe, $\eta(x=45 \mathrm{~m}, t) \geq z$. We track the wave packet backward in space and look at its shape at earlier points in the channel. This allows us to build a collection of extreme events and monitor their precursors. In Fig. 3(a), we show two extreme 

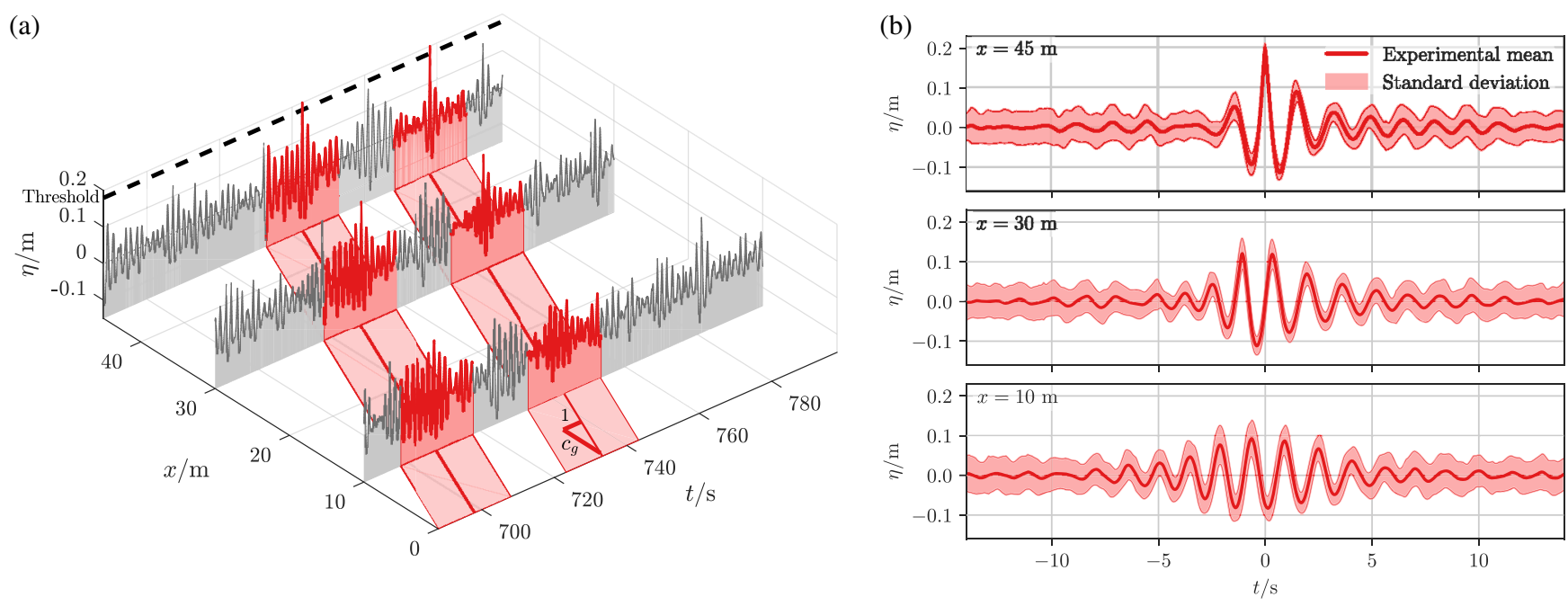

FIG. 3. (a) Extreme wave event selection. At $x=45 \mathrm{~m}$, we monitor the temporal maximum of the experimental data series of $\eta$, record events reaching above a given threshold, and monitor the evolution of these events at probes located earlier in the channel. This is done within an observation time window centered at the maximum and following the wave packet with group velocity $c_{g}$; we repeat this for the whole time series to build a collection of extreme events and their evolution. (b) Mean extreme event. The thick line shows the mean extreme event at different points along the channel, the shaded area a 1 standard deviation range around it. The noise-to-signal ratio is small in the focusing region, leading naturally to the question, can we explain the common pathway by which these rogue waves are most likely to arise?

events at $x=45 \mathrm{~m}$ obtained by this procedure, as well as their precursors at $x=30 \mathrm{~m}$ and $x=10 \mathrm{~m}$. We analyze the statistical properties of these extreme events by computing their average shape and the standard deviation around it at the different positions along the channel, obtaining the result shown in Fig. 3(b) for the highly nonlinear case.

\section{THEORETICAL DESCRIPTION OF ROGUE WAVES VIA INSTANTONS OF NLSE}

We now explain how rogue waves can, within the framework of large deviation, be described as instantons, that is, the minimizers of an action functional associated with the nonlinear Schrödinger equation with random initial data that we will use to describe the system's evolution. In the linear case, as we discuss later, this minimization can be done analytically without much effort. When the nonlinear effects matter, however, numerical computations are required to perform the minimization.

\section{A. Model}

To avoid solving fully nonlinear water wave equations that are complicated from both theoretical and computational viewpoints, it is customary to use simplified models such as the nonlinear Schrödinger equation. If we exclude very nonlinear initial data, it is known that NLSE captures the statistical properties of one-dimensional wave propagation to a good degree of accuracy up to a certain time $[1,2,10,44-46]$ and it can be improved upon by using higher-order envelope equations $[47,48]$. Because of their simplicity, NLSE and extensions thereof have been successfully used to explain basic mechanisms such as the modulational instability in water waves. With the aim of capturing leading order effects, rather than describing the full wave dynamics, here we restrict ourselves to the NLSE as a prototype model for describing the nonlinear and dispersive waves in the wave flume. Higher-order models could in principle improve the agreement between the theoretical instantons and the experimental ones, but as demonstrated later, these corrections are negligible in the wave flume experiment.

In the limit of deep-water, small-steepness, and narrowband properties, the evolution of the system is described, to leading order in nonlinearity and dispersion, by the onedimensional NLSE:

$$
\frac{\partial \psi}{\partial x}+2 \frac{k_{0}}{\omega_{0}} \frac{\partial \psi}{\partial t}+i \frac{k_{0}}{\omega_{0}^{2}} \frac{\partial^{2} \psi}{\partial t^{2}}+2 i k_{0}^{3}|\psi|^{2} \psi=0
$$

The NLSE describes the change of the complex envelope $\psi \equiv \psi(x, t)$ that relates to the surface elevation via the Stokes series truncated at second order:

$$
\eta=|\psi| \cos (\theta)+\frac{1}{2} k_{0}|\psi|^{2} \cos (2 \theta)+O\left(k_{0}^{2}|\psi|^{3}\right),
$$

where $\theta=k_{0} x-\omega_{0} t+\beta$ and $\beta$ is the phase of $\psi$. In this expression the second-order term can be neglected when the field amplitude $|\psi|$ is small-this is the case near the wave maker at $x=0$, where we will specify initial conditions for the NLSE Eq. (6). However, this 
second-order correction is important when $|\psi|$ becomes large, i.e., when rogue waves develop.

The NLSE Eq. (6) is written as an evolution equation in space (rather than in time) in order to facilitate the comparison with experimental data which are taken along the spatial extend of the flume. Consistent with the wave generator located at $x=0$, we specify $\psi(x=0, t)=\psi_{0}(t)$ as the initial condition for Eq. (7), which we take to be a Gaussian random field with a covariance whose Fourier transform is related to the JONSWAP spectrum Eq. (3). Specifically, we set

$$
\psi_{0}(t)=\int_{-\infty}^{\infty} e^{i \omega t} \hat{\psi}_{0}(\omega) d \omega,
$$

with $\hat{\psi}_{0}(\omega)$ Gaussian with mean zero and covariance

$$
\begin{aligned}
& \left\langle\hat{\psi}_{0}(\omega) \overline{\hat{\psi}}_{0}\left(\omega^{\prime}\right)\right\rangle=C\left(\omega-\omega_{0}\right) \delta\left(\omega-\omega^{\prime}\right), \\
& \left\langle\hat{\psi}_{0}(\omega) \hat{\psi}_{0}\left(\omega^{\prime}\right)\right\rangle=\left\langle\overline{\hat{\psi}}_{0}(\omega) \overline{\hat{\psi}}_{0}\left(\omega^{\prime}\right)\right\rangle=0,
\end{aligned}
$$

where the bar denotes complex conjugation and $C(\omega)=$ $C(-\omega)$ is the JONSWAP spectrum defined in Eq. (3). Since, to first order,

$\eta_{0}(t)=\frac{1}{2}\left[\psi_{0}(t) e^{-i \omega_{0} t}+\bar{\psi}_{0}(t) e^{i \omega_{0} t}\right]+O\left(k_{0}\left|\psi_{0}\right|^{2}\right)$,

a direct calculation reported in the appendix shows that, to that order, $\eta_{0}(t)$ is Gaussian with mean zero and covariance $C(\omega)$. Note that in our setup the initial $\psi_{0}(t)$ is the only source of randomness in the model. That is, we evolve $\psi_{0}(t)$ in space by the NLSE, and look for solutions $\psi(x, t)$ whose elevation $\eta(x, t)$ exceeds the threshold $z$ at spatial position $x=L$, i.e., satisfy $\eta(L, t) \geq z$ for some $t \geq 0$ (using temporal invariance we will later designate $t=0$ to be the point in time of the extreme event).

The NLSE Eq. (6) is Hamilton's equation $i\left(\partial_{x}+\right.$ $\left.\left(2 k_{0} / \omega_{0}\right) \partial_{t}\right) \psi=\delta H / \delta \bar{\psi}$ associated with the Hamiltonian $H=H_{\text {lin }}+H_{\text {nl }}$, with

$H_{\text {lin }}=-\frac{k_{0}}{\omega_{0}^{2}} \int_{-\infty}^{\infty}\left|\partial_{t} \psi\right|^{2} d t, \quad H_{\mathrm{nl}}=k_{0}^{3} \int_{-\infty}^{\infty}|\psi|^{4} d t$.

In order to quantify the magnitude of the nonlinearity of the wave field, we use the ratio $\epsilon$ between the nonlinear energy $H_{\mathrm{nl}}$ and the free-particle linear energy $H_{\text {lin. }}$. To this end, we use dimensional analysis to estimate $\left|\partial_{t} \psi\right|^{2}=O\left(\Omega^{2} H_{s}^{2}\right)$ and $|\psi|^{4}=O\left(H_{s}^{4}\right)$, where averaged wave height $H_{s}$ and the characteristic frequency $\Omega$ are defined in Eqs. (4) and (5), respectively. This gives

$$
\epsilon=\frac{H_{\mathrm{nl}}}{H_{\mathrm{lin}}}=\left(\frac{\omega_{0}}{\Omega} k_{0} H_{s}\right)^{2} .
$$

The values of $\epsilon$ obtained this way are given in Table I for the three regimes analyzed: quasilinear, intermediate, and highly nonlinear. We stress that other definitions of the nonlinearity parameter are possible, differing by a constant factor - the important information is the relative magnitude of $\epsilon$ in the different regimes. We also stress that the values of $\epsilon$ are used to interpret the results, but the instanton calculations described next in Sec. IV B are performed in the same way for all values of $\epsilon$.

\section{B. Large deviation theory and instanton calculus}

Our analytical and computational descriptions of rare events rely on instanton theory. Developed originally in the context of quantum chromodynamics [13], at its core lies the realization that the evolution of any stochastic system, be it quantum or classical, reduces to a well-defined (semiclassical) limit in the presence of a small parameter. Concretely, the simultaneous evaluation of all possible realizations of the system subject to a given constraint results in a (classical or path) integral whose integrand contains an action functional $S(\psi)$. The dominating realization can then be obtained by approximating the integral by its saddle point approximation, using the solution to $\delta S\left(\psi^{*}\right) / \delta \psi=0$. This critical point $\psi^{*}$ of the action functional is called the instanton, and it yields the maximum likelihood realization of the event. This conclusion can also be justified mathematically within large deviation theory.

Specifically, we are interested in the probability

$$
P_{L}(z) \equiv \mathbb{P}(\eta(L, 0) \geq z)
$$

i.e., the probability of the surface elevation at position $L$ at an arbitrary time $t=0$ exceeding a threshold $z$. This probability can in principle be obtained by integrating the distribution of the initial conditions over the set

$$
\Lambda(z)=\left\{\psi_{0}: \eta(L, 0) \geq z\right\},
$$

i.e., the set of all initial conditions $\psi_{0}$ at the wave maker $x=0$ that exceed the threshold $z$ further down the flume at $x=L$. Since the initial field $\psi_{0}(t)$ is Gaussian, consistent with Eq. (9) the probability Eq. (13) can therefore be formally written as the path integral

$$
P_{L}(z)=Z^{-1} \int_{\Lambda(z)} \exp \left(-\frac{1}{2}\left\|\psi_{0}\right\|_{C}^{2}\right) D\left[\psi_{0}\right],
$$

where $Z$ is a normalization constant, and we defined

$$
\left\|\psi_{0}\right\|_{C}^{2}=\int_{-\infty}^{\infty} \frac{\left|\hat{\psi}_{0}(\omega)\right|^{2}}{C\left(\omega-\omega_{0}\right)} d \omega,
$$

where $\hat{\psi}_{0}(\omega)=1 /(2 \pi) \int_{-\infty}^{\infty} \psi_{0}(t) e^{-i \omega t} d t$ is the Fourier transform of $\psi_{0}(t)$. The functional integral Eq. (15) can be given a precise mathematical meaning in several ways. For example, we can project the initial field onto finitely many modes, in which case Eq. (15) reduces to a regular integral over these modes. However, even if we were to 
perform this projection, the integration is hard to perform in practice. This is because the set $\Lambda(z)$ defined in Eq. (14) has a very complicated shape in general, that depends nontrivially on the nonlinear dynamics of Eq. (6) since it involves the field at $x=L>0$ down the flume rather than $x=0$. One way around this difficulty is to estimate the integral Eq. (15) via Laplace's method. This strategy is the essence of large deviation theory, or, equivalently, instanton calculus, and it is justified for large $z$, when the probability of the set $\Lambda(z)$ is dominated by a single $\psi_{0}$ contributing most to the integral (see Refs. [32,49]). The optimal condition leads to the constrained minimization problem,

$$
\frac{1}{2} \min _{\psi_{0} \in \Lambda(z)}\left\|\psi_{0}\right\|_{C}^{2} \equiv I_{L}(z)
$$

and gives the large deviation estimate for Eq. (13),

$$
P_{L}(z) \asymp \exp \left[\left(-I_{L}(z)\right],\right.
$$

where the symbol $\asymp$ means asymptotic logarithmic equivalence; i.e., the ratio of the logarithms of the two sides tends to 1 as $z \rightarrow \infty$, or, in other words, the exponential portion of both sides scales in the same way with $z$. Intuitively, the estimate Eq. (18) says that, in the limit of extremely strong (and unlikely) waves, their probability is dominated by their least unlikely realization, the instanton.

In practice, the constraint $\eta(L, 0) \geq z$ can be imposed by adding a Lagrange multiplier term to Eq. (17), and it is easier to use this multiplier as a control parameter and simply see a posteriori what value of $z$ it implies. Concretely, we perform for various values of $\lambda$ the minimization

$$
\min _{\psi_{0}}\left[\frac{1}{2}\left\|\psi_{0}\right\|_{C}^{2}-\lambda \eta(L, 0)\right] \equiv S_{L}(\lambda)
$$

over all the possible realizations of $\psi_{0}$ (without constraint). The minimizer $\psi_{0}^{\star}(\lambda)$ of this optimization problem gives the following parametric representation of $I_{L}(z)$ versus $z$ :

$$
\begin{aligned}
I_{L}[z(\lambda)] & =\frac{1}{2}\left\|\psi_{0}^{\star}(\lambda)\right\|_{C}^{2}, \\
z(\lambda) & =\eta(L, 0)=|\psi(L, 0)|\left\{1+\frac{1}{2} k_{0}|\psi(L, 0)|\right\},
\end{aligned}
$$

where the last equivalence uses the second order of the Stokes series Eq. (7) at $\theta=0$. It is easy to see from Eqs. (17) and (19) that $S_{L}(\lambda)$ is the Legendre transform of $I_{L}(z)$ since

$$
\begin{aligned}
S_{L}(\lambda) & =\sup _{z \in \mathbb{R}}\left[\lambda z-I_{L}(z)\right] \\
& =\sup _{z \in \mathbb{R}}\left(\lambda z-\frac{1}{2} \inf _{\psi_{0} \in \Lambda(z)}\left\|\psi_{0}\right\|_{C}^{2}\right) .
\end{aligned}
$$

It is clear from Eq. (18) that the stochastic sampling problem is replaced by a deterministic optimization problem, which we solve numerically as explained next. The trajectory initiated from the minimizer $\psi_{0}^{*}$ of the action will be referred to as the instanton trajectory, and in the following we compare it to trajectories obtained from the experiment.

\section{Numerical aspects}

In practice, we perform the minimization Eq. (19) by numerical gradient descent in the space of the initial condition $\psi_{0}$, the gradient being computed by the adjoint formalism. Consequently, for each iteration of the descent, the NLSE Eq. (6) needs to be solved up to $x=L$ for the envelope $\psi$ and its adjoint equation for the adjoint field $\tilde{\psi}$. The equation is solved in a time domain of width $75 \mathrm{~s}$, much larger than the correlation time of the wave field (of the order of $10 \mathrm{~s}$ ), with periodic boundary conditions in time. The domain is discretized on a lattice of $2^{11}$ equally spaced points. Combined with a cutoff of the initial spectrum at small amplitude, this leads to $M=89$ modes of the JONSWAP being relevant for the initial data, as depicted in Fig. 2. Equation (6) is numerically integrated in space by means of a pseudospectral exponential timedifferencing method ETDRK2, with a spatial increment of $0.1 \mathrm{~m}$. More details of the numerical procedure can be found in Ref. [32].

The minimizer $\psi_{0}^{\star}$ of Eq. (19) identifies the most likely realization over the distribution of wave shapes at the wave generator which, evolving deterministically via the NLSE, reaches a size $\eta(L, 0) \geq z$. As a saddle point approximation of the corresponding action, $\psi^{\star}(z)$ can be considered the instanton of the problem. Here, the large value of $z$ plays the role of the limiting parameter for the large deviation principle Eq. (18). Thus, the instanton of size $z$ is expected to represent all of the extreme events $\eta(L, 0) \geq z$ to leading order in $z$. Because of this key property, the instanton is the natural object for the characterization of the extreme wave events. Note that the knowledge of the instanton configuration itself can be used as an ingredient for advanced rare event sampling techniques, such as importance sampling and hybrid Monte Carlo approaches [50]. For the purpose of this paper, we restrict our analysis to the comparison of the instanton to the conditioned experimental measurements.

\section{VALIDATION OF THE INSTANTON DESCRIPTION}

In Fig. 4, we compare the evolution of rogue waves observed in the experiment and averaged over many realizations to that of the instanton, both constrained at $x=45 \mathrm{~m}$. In all cases the instanton tracks the dynamics of the averaged wave very closely during the whole evolution. Moreover, in the focusing region the standard deviation around the mean is small, especially toward the end of the 

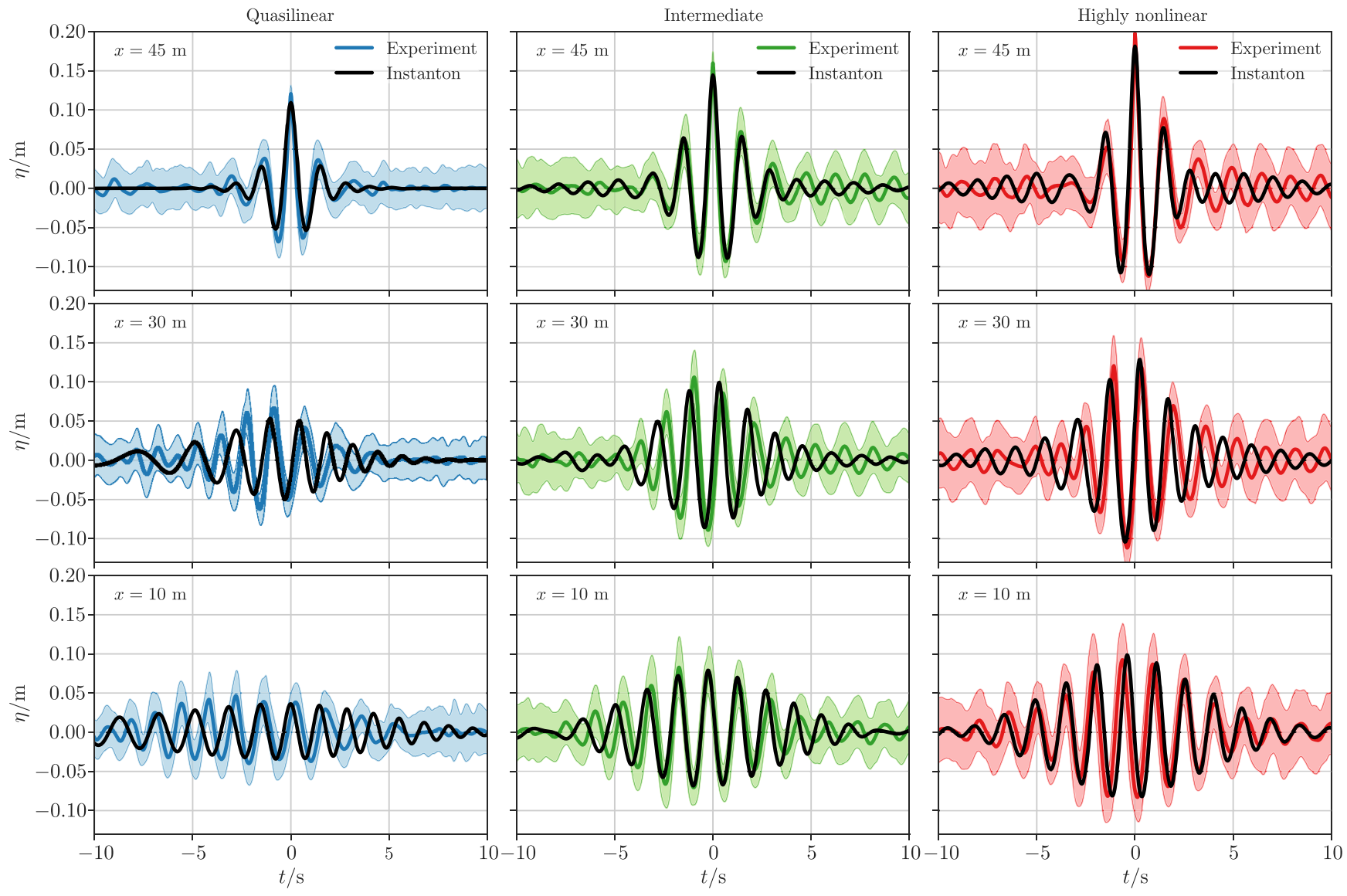

FIG. 4. Experimental validation of the instanton. Snapshots of the instanton during its evolution along the channel (black lines) are compared to the mean and standard deviation of the experimental rogue wave (color lines), for different regimes of nonlinearity. The instanton prediction agrees with the experimental mean across all regimes, and captures the whole evolution along the channel. This confirms that typical rogue waves are well represented by instantons, and the typical extreme events collapse onto the most likely event with only small fluctuations around it.

evolution. This observation in itself is a statement that indeed all of the rogue waves such that $\eta(L, 0) \geq z$ resemble the instanton plus small random fluctuations. The instanton approximation shows excellent agreement not only across different degrees of nonlinearity (and therefore substantially different physical mechanisms), but also captures the behavior of precursors earlier along the channel.

In Fig. 5, the envelope evolution of a single realization of a rogue wave is compared to the instanton evolution at multiple locations, in the highly nonlinear case. In the focusing region the experimental sample shares with the instanton the same overall structure, which is needed to allow it to reach an extreme size.

It is worth stressing that the instanton approach captures both the linear and the fully nonlinear cases, unlike previous theories that could describe each of these regimes individually but not both. To make that point, in the next two sections we compare the predictions of our approach to those of the quasideterministic and semiclassical theories that hold in the dispersive and nonlinear regimes, respectively.

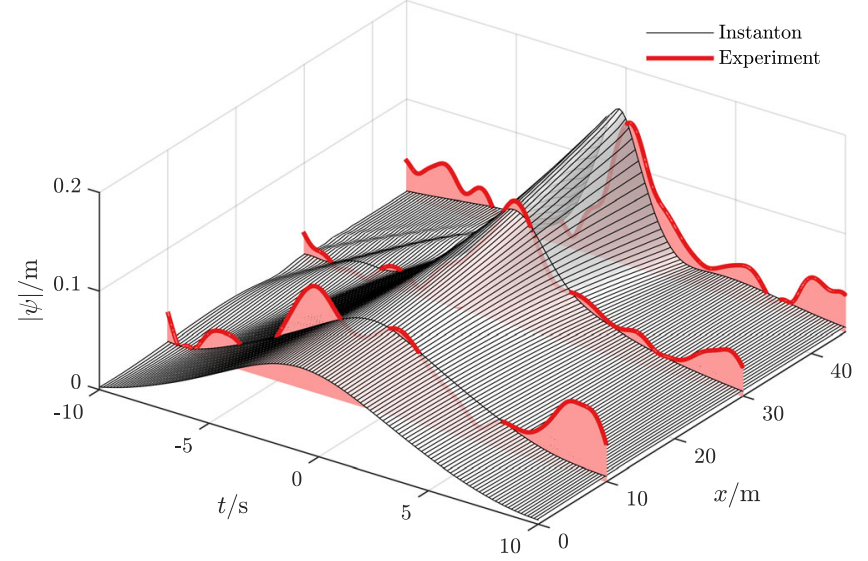

FIG. 5. Agreement of the instanton with individual extreme events. The evolution of a single realization of an extreme wave (red lines) is reasonably approximated by the instanton evolution (black and white surface), here for a sample of the highly nonlinear dataset. In order to capture the focusing pattern in an essential way, the envelope $|\psi|$ is plotted instead of the surface elevation $\eta$ to remove carrier-frequency oscillations. 


\section{A. Comparison to linear theory}

In the linear case, i.e., when the field $\psi(x, t)$ is Gaussian and stationary, the shape of an envelope time series with a large local maximum in $t=0$ is expected to be given by the covariance of the wave field, i.e., the inverse Fourier transform of the spectrum. This is a well-established result in probability [17]. In the oceanographic context, the result was rediscovered in the 1990s [18] and subsequently tested for some real quasi-Gaussian wave records in the ocean [51], also accounting for second-order Stokes corrections [52]. A core result of the theory is the prediction that conditioning the surface elevation to have a large maximum, the expected shape of the water surface is given by the covariance of the wave field, i.e., the inverse Fourier transform of the spectrum. The theory is often referred to as the theory of quasideterminism, which hereafter we name the linear theory for simplicity. In our case, such a prediction is justified if the nonlinear focusing effects are small so that the statistics stay close to Gaussian along the tank, as in the quasilinear set. Then, conditioning on a temporal maximum of $\eta(L, 0)$ at $x=L$, we can compute the history of the wave packet by evolving NLSE backward in space. In Fig. 6(a), this linear prediction is plotted in comparison with the envelope of the averaged rogue wave for the quasilinear set. A good agreement is observed at all spatial points considered. Moreover, the theoretical instanton found through the optimization procedure reduces perfectly to the linear prediction, proving that such a result is included in the instanton theory and represents its limiting linear case. (a)
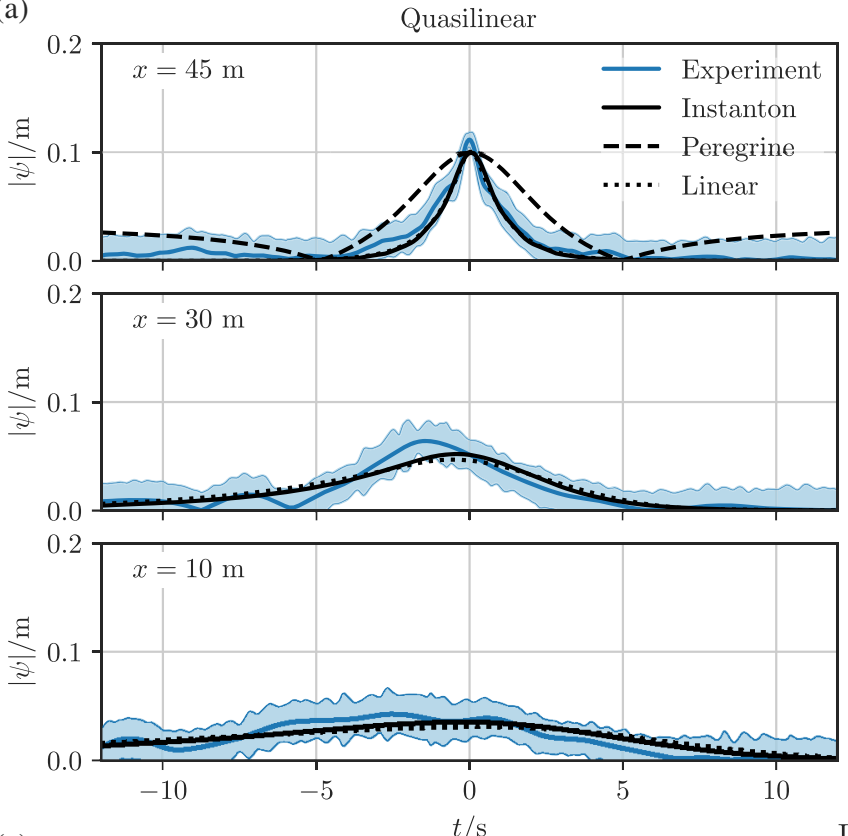

(c)

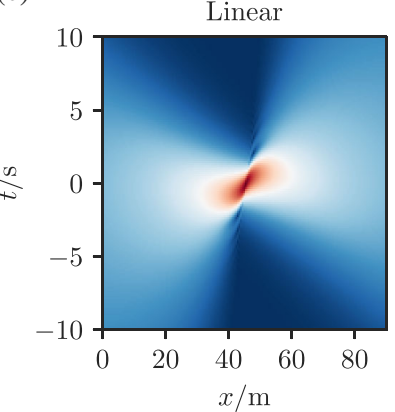

$t / \mathrm{s} \quad$ Quasilinear

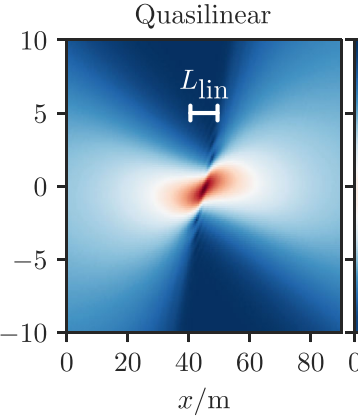

(b)
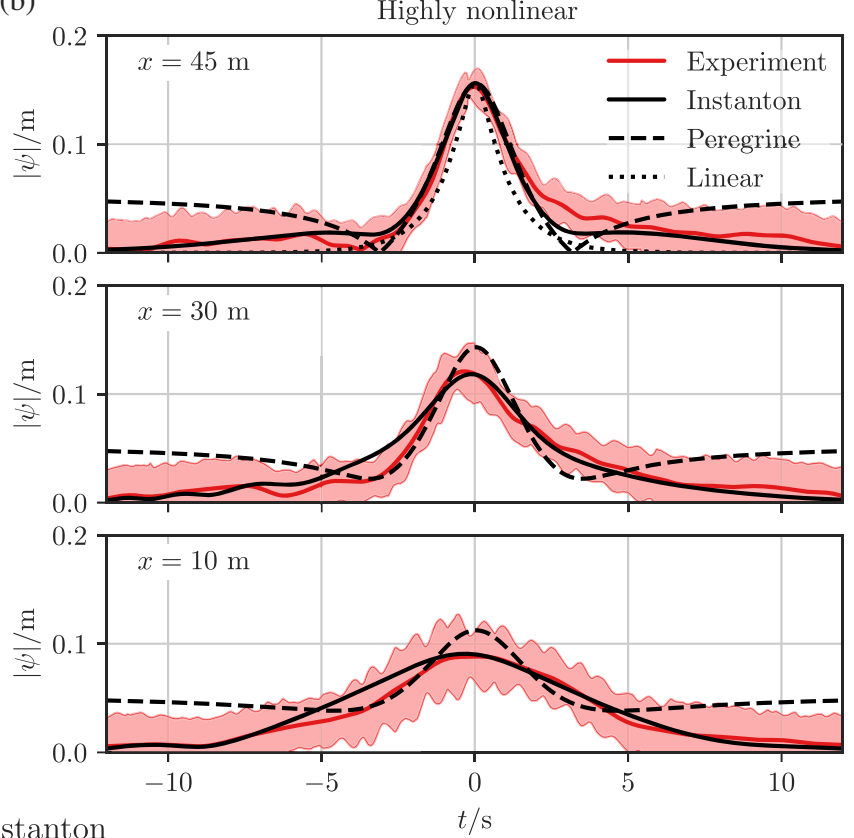

intermediate

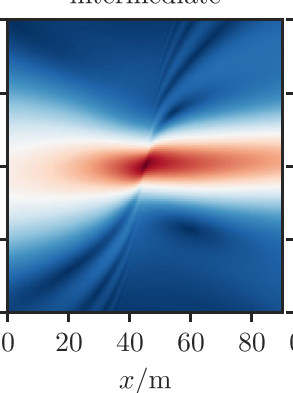

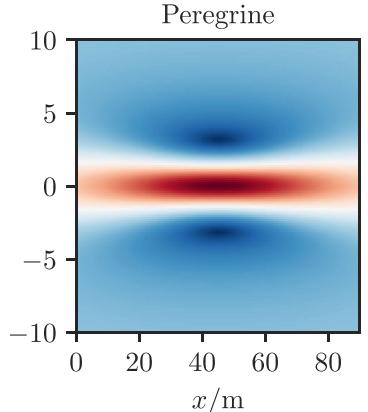

FIG. 6. Comparison of the instanton to the predictions of the theory of quasideterminism and the semiclassical theory. (a) The quasilinear instanton converges to the linear prediction, correctly reproducing the rogue waves averaged over the experiments. (b) The highly nonlinear instanton evolution closely follows the averaged rogue wave and converges locally to a Peregrine soliton around its space-time maximum, as predicted by the semiclassical theory, and reproduced by the instanton. The linear prediction instead fails, especially around the maximum. (c) The contour plots show agreement with the two limiting theories and recover the respective dominant length scales. In the linear limit, dominated by dispersion, the rogue waves arise and decay very rapidly. On the contrary, in the semiclassical limit, where nonlinear effects are prevalent, the Peregrine-like structure of the extreme event is persistent, with a very slow decay. The rogue waves in intermediate regimes display both linear and nonlinear features, as shown in the central panel. 


\section{B. Nonlinear regime and Peregrine solitons}

At the opposite end, in the nonlinear regime, it was recently shown [19] that in the zero-dispersion (semiclassical) regime of the NLSE any single localized pulse on a vanishing background leads locally to the emergence of a Peregrine soliton. By scale invariance of the NLSE, such a regime can be attained whenever an initial condition is characterized by large enough wave groups for which the nonlinear term dominates over the dispersive one. In fiber optics [53,54], emerging Peregrine-like structures have been observed out of a random background. For the highly nonlinear case, in Fig. 6(b) we compare the instanton and the Peregrine soliton reaching the same maximal height $z$ at $x=45 \mathrm{~m}$, finding that in the focusing region the two converge to the same shape, which is also closely followed by the envelope of the experimental averaged rogue wave. Looking at the event precursor at earlier $x$, instead, we notice that the experimental mean wave stays close to the instanton, while it gradually deviates from the Peregrine soliton. Thus, it appears that the instanton captures the mechanism underlying the rogue wave events also when nonlinearity rules over dispersion, tending locally to the Peregrine soliton around the maximal focusing point, consistently with the regularization of the gradient catastrophe [19].

\section{Unified picture of rogue waves}

A useful quantification of the effective mechanisms of rogue wave creation can be obtained by looking at the length scales at play. The linear length of dispersion is given by $L_{\text {lin }}=\omega_{0}^{2} /\left(k_{0} \Omega^{2}\right)$, while the characteristic length associated with the Peregrine soliton is $L_{\mathrm{Per}}=\sqrt{L_{\mathrm{lin}} L_{\mathrm{nl}}}$ [55], where $L_{\mathrm{nl}}=8 /\left(k_{0}^{3} H_{s}^{2}\right)$ is the nonlinear length of modulational instability. These length scales are clearly visible in space-time contours of the amplitude shown in Fig. 6(c). In the linear and quasilinear regimes, the wave packet has a characteristic length around $L_{\text {lin }} \simeq 9 \mathrm{~m}$. Thus, we can state that linear superposition dominates and the expected mechanism leading to the extreme event is the linear dispersion of a coherent wave packet. The quasilinear instanton evolution is almost indistinguishable from the linear approximation. On the other hand, the extent of the structures in the highly nonlinear case agrees with the length $L_{\mathrm{Per}} \simeq 65 \mathrm{~m}$. The dynamics of the highly nonlinear instanton clearly converges to the Peregrine dynamics near the space-time point of maximal focusing, and reproduces the characteristic isolated "dips" of the amplitude observed around the extreme event. Figure 6(c) highlights the sharp difference between the rapidly evanescent linear rogue waves and the more persistent nonlinear ones. Quite strikingly, the instanton is able to interpolate between those two limiting regimes, as evidenced by the intermediate instanton in Fig. 6(c), which displays features of both the linear theory and the Peregrine soliton. Summarizing, the instanton predicts the shape of rogue waves experimentally observed in the tank across all parameter regimes.

\section{Probability estimates from LDT}

The analysis so far has addressed the mechanism of rogue wave formation, and compared the most likely evolution into an extreme wave, as predicted by the instanton, to the observed events measured in the experiment. Since the instanton formalism is based on probability theory and large deviations, it also allows us to deduce the tail scaling of the extreme event probability itself via Eq. (18). Indeed, it was shown in Ref. [49] that the LDT prediction for the tail of the probability density functions (PDFs) matches very well those obtained by brute-force Monte Carlo simulations using NLSE. In the context of actual experiments, the situation is more complicated. Despite the large amount of data collected in the experiments, the far tail of the PDF of the surface elevation is characterized by a natural cutoff related to the phenomenon of wave breaking, visually observed during the experiments in the nonlinear regimes. The NLSE itself misses such effect that lowers the probability to observe rogue wave in experiments, especially in the highly nonlinear regime. As a result, the predictions we can make about the PDFs of rogue waves are less accurate than those about their shape. In Fig. 7, we plot the LDT predictions for the PDF of the surface elevation, $\rho_{L}(z)=-P_{L}^{\prime}(z)$, in the intermediate regime at three spatial points with $L=10,30$, and $45 \mathrm{~m}$ away from the wave maker, and compare them with the experimental PDFs. While the agreement is reasonable past the height threshold for rogue waves, and confirms the expected nonlinear tail fattening [41,42], it is difficult to quantify how accurate these results are because of the problems mentioned earlier.

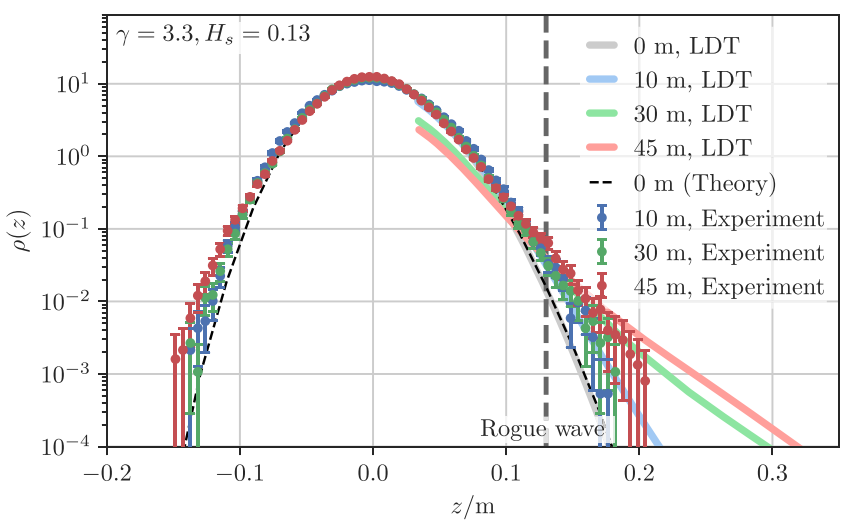

FIG. 7. Comparison of the PDF of the surface elevation $\rho(z)=$ $-P^{\prime}(z)$ obtained by binning the data from the experiments and the LDT estimates from Eq. (18), showing good agreement in the rogue wave regime (right-hand side of the vertical dashed line, indicating the conventional rogue wave threshold $z=H_{s}$ ). The figure refers to the intermediate regime. The blue, green, and red colors indicate data collected at the probes 10, 30, and $45 \mathrm{~m}$ away from the wave maker, respectively. 


\section{CONCLUSIONS}

Starting with the pioneering works in Refs. [56-58], it has been recognized that nonlinear focusing effects may play an important role in the formation of rogue waves. Since then, exact solutions of the NLSE, like, for example, the Peregrine solution, have been reproduced in controlled lab experiments [46,59] and by now are considered as prototypes of rogue waves. In random wave fields, however, our understanding of the development of rogue waves remains more limited. In strongly nonlinear conditions (semiclassical limit), assuming a one-dimensional propagation described by the NLSE, it has been shown [20] that a localized initial condition leads to the development of extreme waves that can be locally fitted to the Peregrine solution of the NLSE. While this fit may suggest the internal mechanism leading to rogue waves in long-crested, narrow-banded deep seas (neglecting other effects such as bathymetry, interactions with sea currents, multimodality, etc., which may also play a significant role in particular situations), it says nothing about their likelihood. Such information is instead intrinsically contained within the instanton framework, allowing for estimates such as in Fig. 7. To what extent these nonlinear effects are at work in real directional sea states is also a difficult question $[8,9,60]$, in part because of the uncertainty in the measurements of the directional wave spectrum, especially close to its peak. If the sea state conditions are not prone for the development of such nonlinear waves, linear dispersion may still be the dominant one for generating rogue waves [8]. This idea is at the core of the theory of quasideterminism (also known as NewWave theory) that was developed in the early 1970s to describe rogue waves in this linear regime $[17,18]$; it allows one to determine the shape of the most extreme wave and relate it to the autocorrelation function. The two, apparently incompatible, mechanisms of formation of rogue waves, i.e., the nonlinear focusing and the linear superposition, have led to many debates among different groups of research.

Here we have proposed a unifying framework based on large deviation theory and instanton calculus that is capable to describe with the same accuracy the shape of rogue waves that result from either a linear superposition or a nonlinear focusing mechanism. In the limit of large nonlinearity, the instantons closely resemble the Peregrine soliton used, e.g., in Refs. [19,20] to describe extreme events, but with the added bonus that our framework predicts their likelihood; in the limit of linear waves, the instanton reduces to the autocorrelation function as obtained in Refs. [17,18]. A smooth transition between the two limiting regimes is also observed, and these predictions are fully supported by experiments performed in a large wave tank with different degrees of nonlinearity. These results were obtained for one-dimensional propagation, but there are no obstacles to apply the approach to two horizontal dimensions, which may finally explain the origin and shape of rogue waves in different setups, including the ocean.

\section{ACKNOWLEDGMENTS}

M. O. has been funded by Progetto di Ricerca d'Ateneo CSTO160004. M. O. and G.D. were supported by the "Departments of Excellence 2018-2022" Grant awarded by the Italian Ministry of Education, University and Research (MIUR) (L.232/2016). E. V.-E. was supported by National Science Foundation (NSF) Materials Research Science and Engineering Center Program Grant No. DMR-1420073, and by NSF Grant No. DMS-1522767. M. O. and E. V.-E. were supported by Simons Collaboration on Wave Turbulence, Grant No. 617006.

\section{APPENDIX: DERIVATION OF EQ. (9)}

Let

$$
\eta_{0}(t)=\frac{1}{2}\left[\left(\psi_{0}(t) e^{-i \omega_{0} t}+\bar{\psi}_{0}(t) e^{i \omega_{0} t}\right],\right.
$$

then, using Eq. (8), this can also be written as

$\eta_{0}(t)=\frac{1}{2} \int_{-\infty}^{\infty}\left[\hat{\psi}_{0}(\omega) e^{i\left(\omega-\omega_{0}\right) t}+\overline{\hat{\psi}}_{0}(\omega) e^{-i\left(\omega-\omega_{0}\right) t}\right] d \omega$.

This implies, using Eq. (9), that

$$
\begin{aligned}
\left\langle\eta(t) \eta\left(t^{\prime}\right)\right\rangle & =\frac{1}{2} \int_{-\infty}^{\infty} C\left(\omega-\omega_{0}\right) \cos \left[\left(\omega-\omega_{0}\right)\left(t-t^{\prime}\right)\right] d \omega \\
& =\int_{0}^{\infty} C(\omega) \cos \left[\omega\left(t-t^{\prime}\right)\right] d \omega
\end{aligned}
$$

which is consistent with Eq. (2).

[1] V. E. Zakharov, Stability of Periodic Waves of Finite Amplitude on the Surface of a Deep Fluid, J. Appl. Mech. Tech. Phys. 9, 190 (1968).

[2] M. Onorato, S. Residori, U. Bortolozzo, A. Montina, and F. Arecchi, Rogue Waves and Their Generating Mechanisms in Different Physical Contexts, Phys. Rep. 528, 47 (2013).

[3] N. Akhmediev, J. M. Dudley, D. Solli, and S. Turitsyn, Recent Progress in Investigating Optical Rogue Waves, J. Opt. 15, 060201 (2013).

[4] H. Bailung, S. K. Sharma, and Y. Nakamura, Observation of Peregrine Solitons in a Multicomponent Plasma with Negative Ions, Phys. Rev. Lett. 107, 255005 (2011).

[5] C. Kharif, E. Pelinovsky, and A. Slunyaev, Rogue Waves in the Ocean (Springer-Verlag, Berlin Heidelberg, 2009).

[6] M. Onorato, T. Waseda, A. Toffoli, L. Cavaleri, O. Gramstad, P. A. E. M. Janssen, T. Kinoshita, J. Monbaliu, N. Mori, A. R. Osborne et al., Statistical Properties of Directional Ocean Waves: The Role of the Modulational 
Instability in the Formation of Extreme Events, Phys. Rev. Lett. 102, 114502 (2009).

[7] T. A. Adcock and P. H. Taylor, The Physics of Anomalous ('Rogue') Ocean Waves, Rep. Prog. Phys. 77, 105901 (2014).

[8] F. Fedele, J. Brennan, S. P. De León, J. Dudley, and F. Dias, Real World Ocean Rogue Waves Explained without the Modulational Instability, Sci. Rep. 6, 27715 (2016).

[9] A. Benetazzo, F. Ardhuin, F. Bergamasco, L. Cavaleri, P. V. Guimarães, M. Schwendeman, M. Sclavo, J. Thomson, and A. Torsello, On the Shape and Likelihood of Oceanic Rogue Waves, Sci. Rep. 7, 8276 (2017).

[10] M. Onorato, A. R. Osborne, M. Serio, and S. Bertone, Freak Waves in Random Oceanic Sea States, Phys. Rev. Lett. 86, 5831 (2001).

[11] R. Rajaraman, Solitons and Instantons: An Introduction to Solitons and Instantons in Quantum Field Theory (NorthHolland, Amsterdam, 1982).

[12] M. I. Dykman, E. Mori, J. Ross, and P. Hunt, Large Fluctuations and Optimal Paths in Chemical Kinetics, J. Chem. Phys. 100, 5735 (1994).

[13] T. Schäfer and E. V. Shuryak, Instantons in QCD, Rev. Mod. Phys. 70, 323 (1998).

[14] G. Falkovich, I. Kolokolov, V. Lebedev, and A. Migdal, Instantons and Intermittency, Phys. Rev. E 54, 4896 (1996).

[15] T. Grafke, R. Grauer, and T. Schäfer, The Instanton Method and Its Numerical Implementation in Fluid Mechanics, J. Phys. A 48, 333001 (2015).

[16] T. Grafke, R. Grauer, T. Schäfer, and E. Vanden-Eijnden, Relevance of Instantons in Burgers Turbulence, Europhys. Lett. 109, 34003 (2015).

[17] G. Lindgren, Local Maxima of Gaussian Fields, Ark. Mat. 10, 195 (1972).

[18] P. Boccotti, Wave Mechanics for Ocean Engineering (Elsevier, New York, 2000), Vol. 64.

[19] M. Bertola and A. Tovbis, Universality for the Focusing Nonlinear Schrödinger Equation at the Gradient Catastrophe Point: Rational Breathers and Poles of the Tritronquée Solution to Painlevé I, Commun. Pure Appl. Math. 66, 678 (2013).

[20] A. Tikan, C. Billet, G. El, A. Tovbis, M. Bertola, T. Sylvestre, F. Gustave, S. Randoux, G. Genty, P. Suret et al., Universality of the Peregrine Soliton in the Focusing Dynamics of the Cubic Nonlinear Schrödinger Equation, Phys. Rev. Lett. 119, 033901 (2017).

[21] M. Onorato, A. Osborne, R. Fedele, and M. Serio, Landau Damping and Coherent Structures in Narrow-Banded $1+1$ Deep Water Gravity Waves, Phys. Rev. E 67, 046305 (2003).

[22] I. Alber, The Effects of Randomness on the Stability of TwoDimensional Surface Wave Trains, Proc. R. Soc. A 363, 525 (1978).

[23] M. Stiassnie, A. Regev, and Y. Agnon, Recurrent Solutions of Alber's Equation for Random Water-Wave Fields, J. Fluid Mech. 598, 245 (2008).

[24] A. Ribal, A. V. Babanin, I. Young, A. Toffoli, and M. Stiassnie, Recurrent Solutions of the Alber Equation Initialized by Joint North Sea Wave Project Spectra, J. Fluid Mech. 719, 314 (2013).
[25] O. Gramstad, Modulational Instability in JONSWAP Sea States Using the Alber Equation, in Proceedings of the 36th ASME International Conference on Ocean, Offshore and Arctic Engineering, 2017 (American Society of Mechanical Engineers, New York, 2017), pp. V07BT06A051V07BT06A051.

[26] A. Athanassoulis, G. Athanassoulis, and T. Sapsis, Localized Instabilities of the Wigner Equation as a Model for the Emergence of Rogue Waves, J. Ocean Eng. Marine Energy 3, 353 (2017).

[27] A. AghaKouchak, D. Easterling, K. Hsu, S. Schubert, and S. Sorooshian, Extremes in a Changing Climate: Detection, Analysis and Uncertainty (Springer Science \& Business Media, Dordrecht, Netherlands, 2012), Vol. 65.

[28] D. Coumou and S. Rahmstorf, A Decade of Weather Extremes, Nat. Clim. Change 2, 491 (2012).

[29] C. B. Field, V. Barros, T. F. Stocker, and Q. Dahe, Managing the Risks of Extreme Events and Disasters to Advance Climate Change Adaptation: Special Report of the Intergovernmental Panel on Climate Change (Cambridge University Press, Cambridge, England, 2012).

[30] T. G. Shepherd, A Common Framework for Approaches to Extreme Event Attribution, Curr. Clim. Change Rep. 2, 28 (2016).

[31] M. A. Mohamad, W. Cousins, and T. P. Sapsis, A Probabilistic Decomposition-Synthesis Method for the Quantification of Rare Events due to Internal Instabilities, J. Comput. Phys. 322, 288 (2016).

[32] G. Dematteis, T. Grafke, and E. Vanden-Eijnden, Extreme Event Quantification in Dynamical Systems with Random Components, SIAM/ASA J. Uncertainty Quantif. 7, 1029 (2019).

[33] P. Glasserman, P. Heidelberger, P. Shahabuddin, and T. Zajic, Multilevel Splitting for Estimating Rare Event Probabilities, Oper. Res. 47, 585 (1999).

[34] S. Juneja and P. Shahabuddin, Rare-Event Simulation Techniques: An Introduction and Recent Advances, in Handbooks in Operations Research and Management Science (North-Holland, Amsterdam, 2006), Vol. 13, p. 291.

[35] F. Cérou and A. Guyader, Adaptive Multilevel Splitting for Rare Event Analysis, Stoch. Anal. Appl. 25, 417 (2007).

[36] C. Giardina, J. Kurchan, V. Lecomte, and J. Tailleur, Simulating Rare Events in Dynamical Processes, J. Stat. Phys. 145, 787 (2011).

[37] J. Tailleur and J. Kurchan, Probing Rare Physical Trajectories with Lyapunov Weighted Dynamics, Nat. Phys. 3, 203 (2007).

[38] E. Vanden-Eijnden and J. Weare, Rare Event Simulation of Small Noise Diffusions, Commun. Pure Appl. Math. 65, 1770 (2012).

[39] M. Farazmand and T. P. Sapsis, A Variational Approach to Probing Extreme Events in Turbulent Dynamical Systems, Sci. Adv. 3, e1701533 (2017).

[40] F. Ragone, J. Wouters, and F. Bouchet, Computation of Extreme Heat Waves in Climate Models Using a Large Deviation Algorithm, Proc. Natl. Acad. Sci. U.S.A. 115, 24 (2018).

[41] M. Onorato, A. R. Osborne, M. Serio, and L. Cavaleri, Modulational Instability and Non-Gaussian Statistics in 
Experimental Random Water-Wave Trains, Phys. Fluids 17, 078101 (2005).

[42] M. Onorato, A. R. Osborne, M. Serio, L. Cavaleri, C. Brandini, and C. T. Stansberg, Extreme Waves, Modulational Instability and Second Order Theory: Wave Flume Experiments on Irregular Waves, Eur. J. Mech. B 25, 586 (2006).

[43] K. Hasselmann, T. P. Barnett, E. Bouws, H. Carlson, D. E. Cartwright, K. Enke, J. A. Ewing, H. Gienapp, D. E. Hasselmann, P. Kruseman et al., Measurements of WindWave Growth and Swell Decay during the Joint North Sea Wave Project (JONSWAP), Deutsches Hydrographisches Institut Technical Report No. 12(A8), 1973.

[44] T. B. Benjamin and J. E. Feir, The Disintegration of Wave Trains on Deep Water Part 1. Theory, J. Fluid Mech. 27, 417 (1967).

[45] N. Akhmediev, J. M. Soto-Crespo, and A. Ankiewicz, Extreme Waves that Appear from Nowhere: On the Nature of Rogue Waves, Phys. Lett. A 373, 2137 (2009).

[46] A. Chabchoub, N. P. Hoffmann, and N. Akhmediev, Rogue Wave Observation in a Water Wave Tank, Phys. Rev. Lett. 106, 204502 (2011).

[47] K. Trulsen, I. Kliakhandler, K. B. Dysthe, and M. G. Velarde, On Weakly Nonlinear Modulation of Waves on Deep Water, Phys. Fluids 12, 2432 (2000).

[48] O. Gramstad and K. Trulsen, Hamiltonian Form of the Modified Nonlinear Schrödinger Equation for Gravity Waves on Arbitrary Depth, J. Fluid Mech. 670, 404 (2011).

[49] G. Dematteis, T. Grafke, and E. Vanden-Eijnden, Rogue Waves and Large Deviations in Deep Sea, Proc. Natl. Acad. Sci. U.S.A. 115, 855 (2018).

[50] G. Margazoglou, L. Biferale, R. Grauer, K. Jansen, D. Mesterházy, T. Rosenow, and R. Tripiccione, Hybrid Monte Carlo Algorithm for Sampling Rare Events in Space-Time Histories of Stochastic Fields, Phys. Rev. E 99, 053303 (2019).

[51] M. A. Tayfun and F. Fedele, Expected Shape of Extreme Waves in Storm Seas, in Proceedings of the 26th ASME
International Conference on Offshore Mechanics and Arctic Engineering, 2007, (American Society of Mechanical Engineers, New York, 2007) pp. 53-60.

[52] F. Fedele and F. Arena, Weakly Nonlinear Statistics of High Random Waves, Phys. Fluids 17, 026601 (2005).

[53] P. Suret, R. El Koussaifi, A. Tikan, C. Evain, S. Randoux, C. Szwaj, and S. Bielawski, Single-Shot Observation of Optical Rogue Waves in Integrable Turbulence Using Time Microscopy, Nat. Commun. 7, 13136 (2016).

[54] A. Tikan, Effect of Local Peregrine Soliton Emergence on Statistics of Random Waves in the 1-D Focusing Nonlinear Schrödinger Equation, arXiv:1905.11938.

[55] R. El Koussaifi, A. Tikan, A. Toffoli, S. Randoux, P. Suret, and M. Onorato, Spontaneous Emergence of Rogue Waves in Partially Coherent Waves: A Quantitative Experimental Comparison between Hydrodynamics and Optics, Phys. Rev. E 97, 012208 (2018).

[56] K. B. Dysthe and K. Trulsen, Note on Breather Type Solutions of the NLS as Models for Freak-Waves, Phys. Scr. T82, 48 (1999).

[57] K. Henderson, D. Peregrine, and J. Dold, Unsteady Water Wave Modulations: Fully Nonlinear Solutions and Comparison with the Nonlinear Schrödinger Equation, Wave Motion 29, 341 (1999).

[58] A. Osborne, M. Onorato, and M. Serio, The Nonlinear Dynamics of Rogue Waves and Holes in DeepWater Gravity Wave Train, Phys. Lett. A 275, 386 (2000).

[59] B. Kibler, J. Fatome, C. Finot, G. Millot, F. Dias, G. Genty, N. Akhmediev, and J. M. Dudley, The Peregrine Soliton in Nonlinear Fibre Optics, Nat. Phys. 6, 790 (2010).

[60] M. Onorato, T. Waseda, A. Toffoli, L. Cavaleri, O. Gramstad, P. A. E. M. Janssen, T. Kinoshita, J. Monbaliu, N. Mori, A. R. Osborne et al., Statistical Properties of Directional Ocean Waves: The Role of the Modulational Instability in the Formation of Extreme Events, Phys. Rev. Lett. 102, 114502 (2009). 